\title{
AGN and star formation activity in local luminous and ultraluminous infrared galaxies (review)
}

\author{
Almudena Alonso-Herrero* ${ }^{* \dagger}$ \\ Instituto de Física de Cantabria, CSIC-Universidad de Cantabria, 39005 Santander, Spain \\ E-mail: aalonsodifca.unican.es
}

The enormous amounts of infrared (IR) radiation emitted by luminous infrared galaxies (LIRGs, $L_{\mathrm{IR}}=10^{11}-10^{12} \mathrm{~L}_{\odot}$ ) and ultraluminous infrared galaxies (ULIRGs, $L_{\mathrm{IR}}>10^{12} \mathrm{~L}_{\odot}$ ) are produced by dust heated by intense star formation (SF) activity and/or an active galactic nucleus (AGN). The elevated star formation rates and high AGN incidence in (U)LIRGs make them ideal candidates to study the interplay between SF and AGN activity in the local universe. In this paper I review recent results on the physical extent of the SF activity, the AGN detection rate (including buried AGN), the AGN bolometric contribution to the luminosity of the systems, as well as the evolution of local LIRGs and ULIRGs. The main emphasis of this review is on recent results from IR observations.

Nuclei of Seyfert galaxies and QSOs - Central engine \& conditions of star formation November 6-8, 2012

Max-Planck-Insitut fÃijr Radioastronomie (MPIfR), Bonn, Germany

\footnotetext{
${ }^{*}$ Speaker.

${ }^{\dagger}$ Augusto G. Linares Senior Research Fellow
} 


\section{Introduction}

Luminous and Ultraluminous infrared (IR) galaxies (LIRGs and ULIRGs) are defined as having IR $(8-1000 \mu \mathrm{m})$ luminosities in the ranges $L_{\mathrm{IR}}=10^{11}-10^{12} \mathrm{~L}_{\odot}$ and $L_{\mathrm{IR}}=10^{12}-10^{13} \mathrm{~L}_{\odot}$, respectively. These immense IR luminosities are produced by dust heated by intense star formation (SF) activity and/or an active galactic nucleus (AGN) (see the review of [31]). The classical optical study of [36] and the later work of [38], among others, showed that the fraction of sources containing an optically identified AGN increases with the IR luminosity of the system. Seyfert nuclei are optically identified in $20 \%-30 \%$ of local LIRGs and up to $~ 58 \%$ of the most luminous ULIRGs (see [38] and references therein).

The triggering of the activity of local ULIRGs and likely the most luminous local LIRGs is mostly driven by major mergers and galaxy interactions. Interestingly, the optical work of [38] found that interacting (U)LIRGs classified as composite (三 powered by both SF and AGN) dominate in the early stages of the interactions, whereas Seyfert nuclei tend to be more common in the later stages. This provides a strong support to the evolutionary scenario where a highly luminous AGN (or even a quasar) appears in the late stages of the interaction after the (U)LIRG phase proposed by [32] almost twenty five years ago. However, at the low luminosity end $\left(L_{\mathrm{IR}}<2-3 \times 10^{11} \mathrm{~L}_{\odot}\right)$ of the local LIRG population major mergers do not dominate in numbers ([30]), and therefore the activity of these LIRGs is likely triggered by other processes, such as minor mergers, fly-by companions, and/or secular evolution.

The IR spectral range, and in particular the mid-IR spectral range $(\sim 5-40 \mu \mathrm{m})$, is rich in continuum and spectral features that can be used to trace both AGN and SF activity with the added advantage that in most of this range the effects of extinction are greatly reduced. While optical studies allowed for tremendous progress in our understanding of the powering mechanisms of (U)LIRGs, they may be missing some of the most obscured AGNs likely to live in these dusty environments. Indeed, the observed deep (in absorption) $9.7 \mu \mathrm{m}$ silicate features of many local ULIRGs betray very embedded nuclear sources (see for instance [34]) with (minimum) optical extinctions of up to $A_{V} \sim 60 \mathrm{mag}$ (if a dust screen model is assumed) or even higher for mixed geometries (see also [12]). Local LIRGs, while not as extreme, still show optical extinctions of up to a few tens of magnitudes (see e.g., [3, 24, 2]). Thus, we need to identify buried AGN in local (U)LIRGs before we attempt to estimate accurately the relative bolometric contributions of SF and AGN activity to their luminosities. In this paper I review briefly recent results using IR observations to quantify the AGN and SF activity contribution to the energetics of local (U)LIRGs. I also discuss the influence of interactions and secular evolution in such activities.

\section{Star Formation Activity in LIRGs and ULIRGs}

Local (U)LIRGs are known to have very high star formation rates (SFR). If all their IR was to be produced only by SF, their IR luminosities would imply SFRs of $17-170 \mathrm{M}_{\odot} \mathrm{yr}^{-1}$ and $170-1700 \mathrm{M}_{\odot} \mathrm{yr}^{-1}$ for LIRGs and ULIRGs, respectively, using the [19] prescription for a Salpeter IMF. The most commonly used mid-IR tracers of SF are the [Ne II]12.81 $\mu \mathrm{m}$ fine structure line sometimes combined with the $[\mathrm{Ne}$ III] $15.56 \mu \mathrm{m}$ line and the polycyclic aromatic features (PAHs). The luminosity of the neon lines is directly proportional to the number of ionizing pho- 
tons ([27, 15]) and thus it probes the most massive and young stars (ages of less than approximately $10 \mathrm{Myr}$ ). Although the PAH features probe longer times scales (a few tens of millions of years), their luminosities are found to correlate well with the IR luminosity of the system in high metallicity starbursts and ULIRGs ([15, 11]) indicating that they can be used to derive SFRs. Additionally, extended continuum mid-IR emission, probing warm dust (typically with temperatures of a few hundred $\mathrm{K}$ ), can be used to estimate the sizes and luminosities of star forming regions.

There is plenty of evidence showing that a large fraction of local LIRGs have SF extending over scales of a few kpc and not only in the nuclear regions. For instance, [24] used the mapping capability of the InfraRed Spectrograph (IRS) on-board Spitzer to observe a small sample of local LIRGs drawn from the volume-limited sample of [3]. They demonstrated that these LIRGs show extended [Ne II], [Ne III], and PAH emission, with the $11.3 \mu \mathrm{m}$ PAH emission appearing more extended than the [Ne II] one (see also [8, 7]). This was explained because the $11.3 \mu \mathrm{m}$ PAH emission can be excited by stars older than those responsible for the neon emission and/or because of the presence of diffuse PAH emission in photo-dissociation regions.

[9] studied the extended mid-IR continuum emission of a large sample of local (U)LIRGs

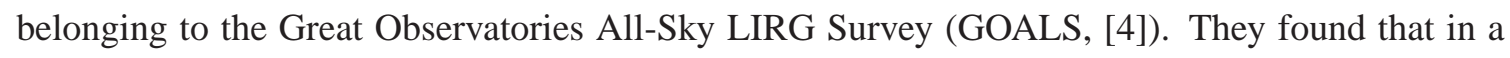
large fraction of local LIRGs more than $50 \%$ of their mid-IR emission arises from circumnuclear regions extending up to scales of about $10 \mathrm{kpc}$ (see also [1]). This is in contrast with the most luminous LIRGs $\left(L_{\mathrm{IR}}>6 \times 10^{11} \mathrm{~L}_{\odot}\right)$ and ULIRGs where most of their mid-IR emission comes from the nuclear regions on scales of less than $1 \mathrm{kpc}$ (see also [33]). As we shall see in Section 3 , this does not necessarily imply that local ULIRGs do not have extended SF (see e.g., [28]), but rather that their mid-IR emission has a large or even a dominant contribution from an AGN.

\section{AGN Activity in LIRGs and ULIRGs}

\subsection{Mid-IR AGN indicators}

The mid-IR spectral range is rich in continuum and line features that can be used to identify and quantify the AGN emission in galaxies. The most direct way to detect an AGN in this range is by detection of fine-structure lines whose ionization potentials are sufficiently high that they can only be excited by AGN-produced photons. Among them, the [Ne v] lines at 14.32 and $24.32 \mu \mathrm{m}$ are the best choice as they are unambiguously produced by AGN (see [12]). However, a caveat to keep in mind is that these [Ne V] lines are not always detected in local bright AGN (see e.g., [25]). Indeed, the [Ne v] detection rate remains relatively low in local LIRGs (approximately 20\%, [26, 2]) and varies between 25\% ([35]) and 30-50\% ([1] ] in local ULIRGs

Other fine-structure lines with intermediate ionization potentials, such as the [O IV] $25.89 \mu \mathrm{m}$ line, while excited by both SF and AGN, they can also be useful when combined with low ionization lines produced by SF (for instance, the [Ne II] line). The [O IV] line has a higher detection rate than the [Ne V] lines in (U)LIRGs, but it is detected in some (U)LIRGs optically classified as star-forming galaxies. Nevertheless, most local LIRGs show [O IV]/[Ne II] ratios similar to those of high metallicity starburst galaxies, with only a small fraction $(\sim 10 \%$, mostly bright Seyfert nuclei) showing ratios comparable to those of pure AGN ([26, 2] ]). ULIRGs on the other hand, show a broader spread in fractional AGN and starburst contributions to the [O IV] line ([11, 35]), indicating higher AGN bolometric contributions as we shall see in Section 3.2 . 


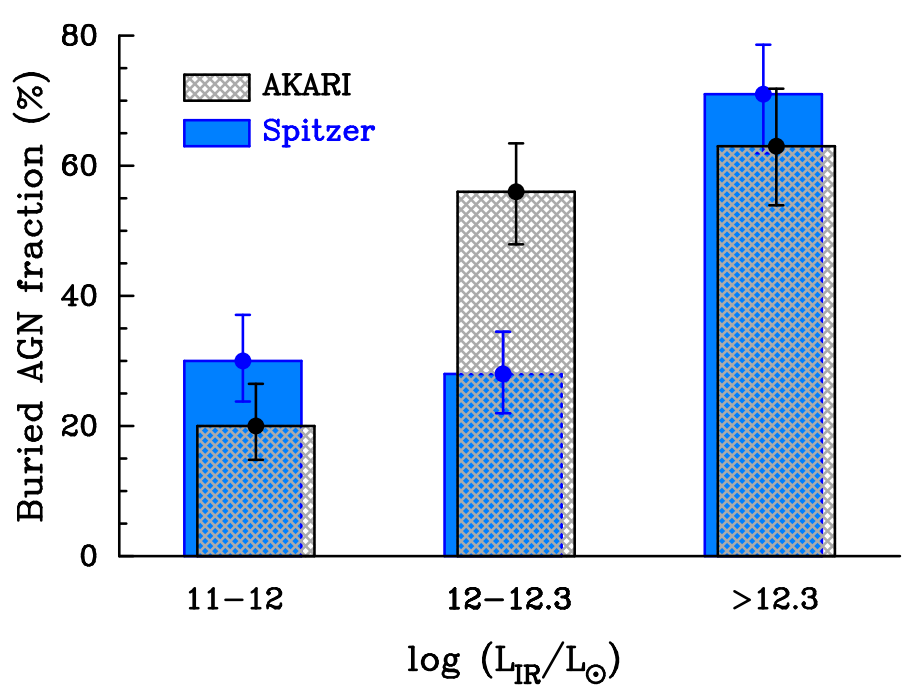

Figure 1: Fraction of optically elusive (i.e., not classified as Seyfert nuclei in the optical) buried AGN for local LIRGs and ULIRGs. Spitzer results for local LIRGs are from [2], whereas the Spitzer results for local ULIRGs and the AKARI results are from [17]. The error bars are the $1 \sigma$ uncertainties of the fractions based on the number of AGN detections relative to the total number of objects in each IR luminosity bin.

As discussed in Section 2 . PAH emission is ubiquitous and remarkably similar in high metallicity starburst galaxies in the local universe ([[]]). Thus, the equivalent widths (EWs) of different PAH features (e.g., at 3.3, 6.2, 7.7, and $11.3 \mu \mathrm{m}$ ) are commonly used to identify AGN especially in composite sources as well as to detect buried AGN (see e.g., [18, 17] and references therein). This is because in the presence of SF, the hot and warm dust continuum produced by an AGN would effectively decrease the EW of PAH features. Also it is even possible that the hard radiation field of AGN might destroy the PAH molecule carriers, although this is still a matter of controversy. Therefore, diagnostic diagrams combining the EW of a PAH feature with for instance the $[\mathrm{O} \mathrm{IV}] /[\mathrm{Ne} \mathrm{II}]$ ratio or the strength of the $9.7 \mu \mathrm{m}$ feature are commonly used to assess the AGN and SF contributions of (U)LIRGs and galaxies in general (see e.g., [12, 34, 11, 35, 26, 13, 26]).

Hinging on the similarity of the PAH emission in starburst galaxies, spectral decomposition methods of Spitzer/IRS spectra have demonstrated to be highly effective to identify AGN and quantify the AGN emission in local (U)LIRGs (see [23, 22, 目) and in high- $z$ IR bright galaxies (e.g., [29, 14]). Using the entire Spitzer/IRS spectral range $(\sim 5-40 \mu \mathrm{m})$ [2] detected an AGN component in half of the volume-limited sample of local LIRGs of [B]. When also adding those objects optically classified as composite but without a mid-IR AGN detection, [2] found that the AGN detection rate in local LIRGs is $60 \%-65 \%$ with no dependence with the IR luminosity of the system. For a sample of local ULIRGs and using the $5-8 \mu \mathrm{m}$ spectral range [22] obtained a similar AGN detection rate (70\%). The AGN detection rate in local (U)LIRGs is much higher than for instance in the optically selected RSA sample (5-16\%, see [21]). This already hints at a close relation between SF and AGN activity in local (U)LIRGs, as we shall discuss in Section $\emptyset$.

As we have seen, IR observations are very effective in identifying AGN in the dusty environments of local (U)LIRGs, even in those cases when the AGN had not been detected optically (also known as optically elusive buried AGN). [17] (and references therein) recently quantified the frac- 


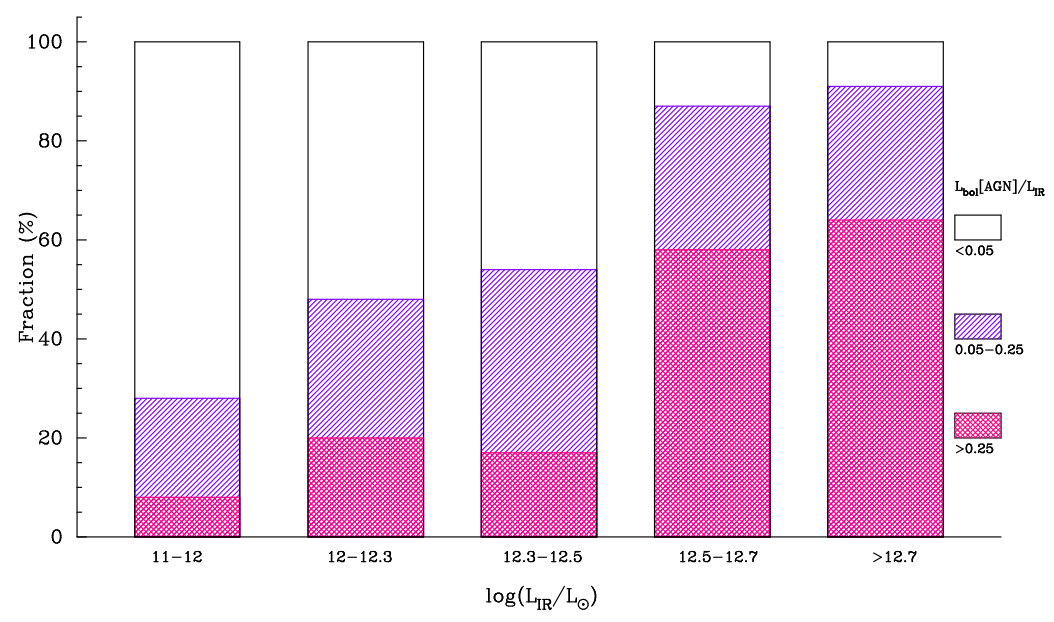

Figure 2: Summary of the fractional AGN contribution to the IR luminosity of the volume-limited sample of local LIRGs of [3, 2 ] and comparison with local ULIRGs of [22]. For each luminosity bin we show three different ranges of AGN to IR luminosity ratios. Adapted from [⿰冫欠].

tion of buried AGN in local (U)LIRGs using both Spitzer mid-IR spectroscopy and AKARI near-IR spectroscopy. Figure 11 summarizes their results using Spitzer and AKARI spectroscopy. We also included in this figure the buried AGN fraction from Spitzer spectroscopy of local LIRGs from [2]. Except for the $\log \left(\mathrm{L}_{\mathrm{IR}} / \mathrm{L}_{\odot}\right)=12-12.3$ bin where there is a discrepancy, the AKARI and Spitzer estimates are consistent with each other. Clearly this fraction increases with the IR luminosity of the system going from $\sim 25 \%$ in LIRGs to about $70 \%$ in the most luminous ULIRGs. This likely reflects the fact that the active nuclei of ULIRGs are much more embedded, since the total AGN fractions of LIRGs and ULIRGs are similar.

\subsection{AGN bolometric contributions}

Although the combined optical+IR AGN detection rate of local LIRGs is only slightly less than that of ULIRGs, it is important to quantify the AGN bolometric contributions in both types of galaxies. One advantage of the spectral decomposition methods is that they can provide an estimate of the AGN bolometric contribution to the IR luminosity of the systems either by using torus models to reproduce the AGN spectral features in the mid-IR (see [2] for details) or by making a bolometric correction at a specified mid-IR rest-frame luminosity of the AGN (as done by [35] and [23, 22]). [2] found that AGN only contribute $5_{-3}^{+8} \%$ of the IR luminosity of local LIRGs. 26] found a similar result for the LIRGs in the GOALS sample. These estimates prove that in local LIRGs the bulk of the IR luminosity is due to SF activity. In local ULIRGs, the AGN contribution is on average $\sim 30 \%-40 \%$ depending on the sample and methods used for the estimates ([35, 22] $)$. We also note here that [35] found that the AGN bolometric contributions to the luminosity of the system of some local ULIRGs can be as high as those of Palomar-Green (PG) quasars. The PG quasars have, however, lower IR luminosities than ULIRGs.

Figure 2 shows the comparison of AGN bolometric contributions of local LIRGs from [河 and ULIRGs from [22] in three ranges of bolometric contributions. As can be seen from this figure, only about $10 \%$ of local LIRGs have significant AGN bolometric contributions (above 25\%). These high AGN bolometric contributions increase noticeably with the IR luminosity of ULIRGs 
(see also [35]) raising to $\sim 60 \%$ for the most luminous ULIRGs. Additionally [35] studied the AGN bolometric contributions in ULIRGs in terms of other properties such as IR colors, optical class, and interaction properties. They found that the AGN contribution is higher in ULIRGs with "warmer" IRAS colors than those with "cool" colors. ULIRGs optically classified as H II tend to show the lowest AGN contributions, while the AGN fractional contributions increase from those classified as Seyfert 2 to the highest contributions for nuclei optically classified as Seyfert 1. Finally, there is a slight tendency for increased AGN contributions at the smallest nuclear separations and latest stages of the interaction.

\section{Evolution of SF and AGN activity in local LIRGs and ULIRGs}

Mergers of gas-rich galaxies are efficient in both producing elevated SFR and transporting gas to the vicinity of the nucleus to allow for black hole $(\mathrm{BH})$ growth. If sufficient matter becomes available very close to the nuclear BH and is accreted, the nucleus of the galaxy will shine with enormous power as an AGN. In parallel, and probably before the fully developed AGN phase, the merger will trigger a high SFR leading to a luminous IR-active phase, either as a luminous IR galaxy LIRG or a ULIRG (see e.g., the simulations of [16]). [32] proposed such an evolutionary scenario between ULIRGs and quasars almost twenty five years ago. [20] (and references therein) proposed an alternative to this merger-driven sequence, where BHs in bulgeless galaxies and in galaxies with pseudobulges probably grow through phases of low level Seyfert-like activity. This growth is likely to be driven stochastically by local processes (secular processes) not associated with major mergers, and thus they would not have a global impact on the host galaxy structure.

Since the triggering of the activity in local ULIRGs and likely also in the most luminous local LIRGs is driven by major mergers, ULIRGs tend to host more luminous AGN (quasar or nearly quasar-like luminosities), slightly more massive $\mathrm{BH}$ and have higher AGN bolometric contributions that local LIRGs ([35, 22, 目, 1]). While the simplicity of the [32] scenario is appealing, several works have argued that some modifications are needed. For instance, based on the scatter of some of the properties of ULIRGs (e.g., AGN contribution, Eddington ratios, obscuration) [35] proposed a revised evolutionary picture where the initial conditions of the interaction would play an important role. Moreover, [10] put forward an evolutionary paradigm of dusty AGN where some ULIRGs do not go through a quasar phase at all.

A number of works (see e.g., [30, 3]) have shown that major mergers are not prevalent in the population of local LIRGs at $L_{\mathrm{IR}} \lesssim 3 \times 10^{11} L_{\odot}$. Therefore, in most local LIRGs the current episodes of star formation activity and $\mathrm{BH}$ growth could be due to other processes such as minor mergers, fly-by companions, and/or secular evolution. Still, the same sequence of events as in ULIRGs appears in LIRGs. [他 recently found that local LIRGs go through a distinct IR-bright star forming phase taking place prior to the bulk of the current BH growth (that is, the AGN phase). In other words, there is a time delay between the peaks of SF and BH growth, as found for optically identified Seyfert galaxies in the local universe ([6, 37]) and is postulated for local ULIRGs.

The author wishes to thank the organizers of the workshop for the invitation to give this review and also to M. Imanishi for sharing his results for Figure 1. This work was supported in part by the Augusto González Linares Program from the Universidad de Cantabria. 


\section{References}

[1] A. Alonso-Herrero et al., Local Luminous Infrared Galaxies. III. Co-evolution of Black Hole Growth and Star Formation Activity?, ApJ in press (2013) [arXiv: 1301 . 4015]

[2] A. Alonso-Herrero, et al., Local Luminous Infrared Galaxies. II. Active Galactic Nucleus Activity from Spitzer/Infrared Spectrograph Spectra, ApJ 744 (2012) 2 [arXiv: 1109.1372 ]

[3] A. Alonso-Herrero, et al., Near-Infrared and Star-forming Properties of Local Luminous Infrared Galaxies, ApJ $\mathbf{6 5 0}$ (2006) 835 [arXiv: astro-ph/ 0606186 ]

[4] L. Armus et al., GOALS: The Great Observatories All-Sky LIRG Survey, PASP 121 (2009) 559 [arXiv:0904.4498]

[5] B. R. Brandl et al., The Mid-Infrared Properties of Starburst Galaxies from Spitzer-IRS Spectroscopy, ApJ 653 (2006) 1129 [arXiv:astro-ph/ 0609024 ]

[6] R. I. Davies et al., A Close Look at Star Formation around Active Galactic Nuclei, ApJ 671 (2007) 1388 [arXiv:0704.1374]

[7] T. Díaz-Santos et al., The Spatial Extent of (U)LIRGs in the Mid-infrared. II. Feature Emission, ApJ 741 (2011) 32 [arXiv: 1107.5958 ]

[8] T. Díaz-Santos et al., A High Spatial Resolution Mid-Infrared Spectroscopic Study of the Nuclei and Star-Forming Regions in Luminous Infrared Galaxies, ApJ 711 (2010a) 328 [arXiv: 1001 . 1871]

[9] T. Díaz-Santos et al., The Spatial Extent of (U)LIRGs in the Mid-infrared. I. The Continuum Emission, ApJ 723 (2010b) 993 [arXiv: 1009.0038 ]

[10] D. Farrah et al., An Evolutionary Paradigm for Dusty Active Galaxies at Low Redshift, ApJ 700 (2009) 395 [arXiv:0905.1956]

[11] D. Farrah et al., High-Resolution Mid-Infrared Spectroscopy of Ultraluminous Infrared Galaxies, ApJ 667 (2007) 149 [arXiv: 0706.0513$]$

[12] R. Genzel et al., What powers Ultraluminous IRAS Galaxies, ApJ 498 (1998) 579 [arXiv:astro-ph/9711255]

[13] A. Hernán-Caballero \& E. Hatziminaoglou, An atlas of mid-infrared spectra of star-forming and active galaxies, MNRAS 414 (2011) 500 [arXiv: 1101 . 4794]

[14] A. Hernán-Caballero et al., Mid-infrared spectroscopy of infrared-luminous galaxies at $z \sim 0.5-3$, MNRAS 395 (2009) 1695 [arXiv: 0902 . 3369]

[15] L. C. Ho, \& E. Keto, The Mid-Infrared Fine-Structure Lines of Neon as an Indicator of Star Formation Rate in Galaxies, ApJ 658 (2007) 314 [arXiv:astro-ph / 0611856 ]

[16] P. F. Hopkins et al., A Cosmological Framework for the Co-Evolution of Quasars, Supermassive Black Holes, and Elliptical Galaxies. I. Galaxy Mergers and Quasar Activity, ApJS 175 (2008) 356 [arXiv:0706.1243]

[17] M. Imanishi et al., AKARI IRC Infrared 2.5-5 $\mu \mathrm{m}$ Spectroscopy of a Large Sample of Luminous Infrared Galaxies, ApJ 721 (2010) 1233 [arXiv:1008.1585]

[18] M. Imanishi et al., A Spitzer IRS Low-Resolution Spectroscopic Search for Buried AGNs in Nearby Ultraluminous Infrared Galaxies: A Constraint on Geometry between Energy Sources and Dust, ApJS 171 (2007) 72 [arXiv:astro-ph/0702136] 
[19] R. C. Jr. Kennicutt, Star Formation in Galaxies Along the Hubble Sequence, ARA\&A 36 (1998) 189 [arXiv:astro-ph/9807187]

[20] J. Kormendy et al., Supermassive black holes do not correlate with galaxy disks or pseudobulges, Nature 469 (2011) 374 [arXiv: 1101 . 3781]

[21] R. Maiolino \& G. H. Rieke, Low-Luminosity and Obscured Seyfert Nuclei in Nearby Galaxies, ApJ 454 (1995) 95

[22] E. Nardini et al., The role of nuclear activity as the power source of ultraluminous infrared galaxies, MNRAS 405 (2010) 2505 [arXiv: 1003.0858 ]

[23] E. Nardini et al., Spectral decomposition of starbursts and active galactic nuclei in 5-84m Spitzer-IRS spectra of local ultraluminous infrared galaxies, MNRAS 385 (2008) L130 [arXiv : 0801 . 4304]

[24] M. Pereira-Santaella et al., Local Luminous Infrared Galaxies. I. Spatially Resolved Observations with the Spitzer Infrared Spectrograph, ApJS 188 (2010) 447 [arXiv: 1004 . 1364]

[25] M. Pereira-Santaella et al., The Mid-infrared High-ionization Lines from Active Galactic Nuclei and Star-forming Galaxies, ApJ 725 (2010) 2270 [arXiv: 1010 . 5129]

[26] A. O. Petric et al., Mid-Infrared Spectral Diagnostics of Luminous Infrared Galaxies, ApJ 730 (2011) 28 [arXiv:1012.1891]

[27] P. F. Roche, D. K. Aitken, C. H. Smith, \& M. J. Ward, An atlas of mid-infrared spectra of galaxy nuclei, MNRAS, 248 (1991) 606

[28] J. Rodríguez-Zaurín et al., VLT-VIMOS integral field spectroscopy of luminous and ultraluminous infrared galaxies. III. The atlas of the stellar and ionized gas distribution, A\&A 527 (2011) A60 [arXiv:1009.0112]

[29] A. Sajina et al., Spitzer Mid-Infrared Spectroscopy of Infrared Luminous Galaxies at z 2. II. Diagnostics, ApJ 664 (2007) 713 [arXiv: 0805 . 0363]

[30] D. B. Sanders \& C. Ishida, Luminous Infrared Galaxies: Optical Morphology and Photometry, Molecular Gas Masses, and Starburst/AGN Activity versus Infrared Luminosity, in ASPC 320 (2004) 230

[31] D. B. Sanders \& I. F. Mirabel, Luminous Infrared Galaxies, ARA\&A 34 (1996) 749

[32] D. B. Sanders et al., Ultraluminous infrared galaxies and the origin of quasars, ApJ 325 (1988) 74

[33] B. T. Soifer et al., High Resolution Mid-Infrared Imaging of Ultraluminous Infrared Galaxies, AJ 119 (2000) 509 [arXiv:astro-ph/9911045]

[34] H. W. W. Spoon et al., Mid-Infrared Galaxy Classification Based on Silicate Obscuration and PAH Equivalent Width, ApJ 654 (2007) 49 [arXiv:astro-ph/0611918]

[35] S. Veilleux et al., Spitzer Quasar and ULIRG Evolution Study (QUEST). IV. Comparison of 1Jy Ultraluminous Infrared Galaxies with Palomar-Green Quasars, ApJS 182 (2009) 628 [arXiv:0905.1577]

[36] S. Veilleux et al., Optical Spectroscopy of Luminous Infrared Galaxies. II. Analysis of the Nuclear and Long-Slit Data, ApJS 98 (1995) 171

[37] V. Wild et al., Timing the starburst-AGN connection, MNRAS 405 (2010) 933 [arXiv: 1002 . 3156]

[38] T.-T. Yuan et al., The Role of Starburst-Active Galactic Nucleus Composites in Luminous Infrared Galaxy Mergers: Insights from the New Optical Classification Scheme, ApJ 710 (2010) 884 [arXiv:0911.3728] 